\title{
The role of MRI in staging and radiotherapy treatment planning in locally advanced nasopharyngeal carcinoma - case report
}

\author{
Ivanovska Hr. ${ }^{1}$, Encheva E. ${ }^{1}$, Iliev G. ${ }^{2}$, Georgiev R. ${ }^{3}$, Chaushev B. ${ }^{4}$, \\ Bochev P. ${ }^{4}$, Yordanova Ts. ${ }^{4}$, Dimitrova E. ${ }^{5}$, Tsonev N. ${ }^{5}$, Shterev I. ${ }^{5}$ \\ 'Radiotherapy Department, St. Marina University Hospital, Varna, Medical University of Varna, Bulgaria \\ ${ }^{2}$ ENT Department, St. Marina University Hospital, Varna, Medical University of Varna, Bulgaria \\ ${ }^{3}$ Department of imaging diagnostics, St. Marina University Hospital, Varna, Medical University of Varna, Bulgaria \\ ${ }^{4}$ Nuclear Medicine Department, St. Marina University Hospital, Varna, Medical University of Varna, Bulgaria \\ ${ }^{5}$ Medical Oncology Department, St. Marina University Hospital, Varna, Medical University of Varna, Bulgaria
}

\begin{abstract}
:
Nasopharyngeal carcinoma (NPC) is a rare disease. The "Golden imaging standard" for primary tumor and regional lymph nodes staging in NPC is MRI- magnetic resonance imaging. Radiotherapy is the main treatment modality in both early and locoregional advanced NPC. Radiotherapy alone is recommended only in T1N0M0 stage NPC, while in higher stages for best treatment results contributes radiotherapy with concomitant chemotherapy. The aim is to report a case of a patients with NPC presented to our department with clinical and MRI data for scull base and brain invasion. Tumor staging included PET/CT scan and MRI, which were used also for treatment planning. The patient was treated with definitive radiotherapy to 64 Gy, concomitantly with weekly Cisplatin. VMAT and IGRT treatment techniques were used. One year later a complete response is observed without late toxicity and grade 2 dry mouth. Using of MRI for initial staging and radiotherapy planning in NPC is very important due to precise findings of the tumor invasion and a correct definition of the treatment volume. MRI staging should be part of the initial staging and follow up algorithm in all patients with NPC.
\end{abstract}

Key words: Nasopharyngeal cancer, radiotherapy, MRI, staging, target volumes contouring, , PET-CT

\section{Introduction}

Nasopharyngeal carcinoma (NPC) is a rare disease. The incidence per year is $0.5-2.0$ cases per 100,000 for the Europe and USA, and higher in countries such as China- 25 per 100,000, where is considered to be endemic. NPC occurs predominantly in males and the most common reported pathogenic factor is Epstein-Barr virus (EBV) infection $(1,2)$. In Bulgaria NPC occurs in similar incidence $0.5 / 100000$ people) (3). Yearly in Bulgaria are diagnosed about 70 patients, most of them in locoregional advanced stage. The "Golden standard" for staging NPC is MRI. It evaluates both $\mathrm{T}$ category of the primary tumor, and $\mathrm{N}$ category - regional lymph nodes status. Radiotherapy is the main treatment modality in both early and locoregional advanced NPC. Radiotherapy alone is recommended only in T1N0M0 stage, while in higher stages for best treatment results contributes radiotherapy with concomitant chemotherapy (4). As MRI best defines the tumor extension and lymph node involvement, it is the preferred imaging mode for contouring the target volumes for irradiation.

The current clinical case demonstrates the importance of MRI for NPC staging and its role for precise treatment planning in locally advanced NPC. 
The most appropriate and recommended radiotherapy technique in NPC is intensity modulated radiation therapy (IMRT). It allows simultaneous delivery of maximum dose in the tumor volume and minimum dose in healthy organs better than 3D conventional RT and contributes to lower rate of early and late toxicity. In the recent years more advanced IMRT technique was introduced volumetric modulated arc therapy (VMAT). VMAT reduces the monitor units delivered in the patients and the treatment time compared to IMRT, which could be beneficial to reduce the dose in healthy tissues and reduce toxicity. For reducing the dose at organ at risk is important to reduce the safety margin around clinical target volume, which is possible when 3D image guidance is used, so called image guided radiotherapy (IGRT). IGRT relies on 3D images obtained in treatment room for verification of tumor and patient position before delivering every fraction. This support the precise and accurate delivery with IMRT and VMAT irradiation techniques.

The aim is to report a case of patient presenting at our department with locoregional advanced NPC with scull base invasion.

\section{Case report}

The repoted patient was 37 years old, male, smoker with complaints of headache, otalgia in right ear, paresthesia in right part of the head and big mass in the right neck. In June 2015 a biopsy was performed at the ENT Department, UH "St.Marina" - Varna, pathology report showed non-keratinizing carcinoma. Diagnostic PET/CT was performed with result for locally advanced NPC, and positive lymph nodes at levels IIA, IIB, IV, VA and VB on the right neck and single lymph node at levels IIA and $\mathrm{B}$ on the left. PET/CT did not reported a scull base invasion, and NPC was staged cT3 cN3 cM0 (fig.1). When patients presented at radiotherapy department for a consultation he reported facial paresthesia and he was further referred to MRI. MRI showed locally advanced NPC, with scull base invasion and brain involvement, next to the right optic nerve and chiasm and brainstem (fig.2). The correct stage of the disease was T4 N3 M0 and at multidisciplinary tumor board meeting, was taken decision for concurrent chemoradiation with curative intent. In June 2015 the patient started radiation treatment at Radiotherapy Department, UH "St. Marina"- Varna with volumetric modulat- ed arc therapy (VMAT) using $6 \mathrm{MV}$ photons, IGRT (cone beam computed tomography). All diagnostic images of the patient MRI and PET-CT were imported in the treatment planning system. Target volumes were defined on fused images between the CT simulator images and MRI images (fig. 3). Due to proximity to critical structures, the gross tumor volume in the nasopharynx and neck received 64Gy in 2Gy daily fractions, while the tolerance doses at surrounding organs atrisk were kept below the constrains. Decision for total dose of $64 \mathrm{~Gy}$ was taken after dosimetric plan evaluation and it is in correlation with benefits and risks for the patient, local tumor control, quality of life and total dose recommendation. The concomitant chemotherapy was given as weekly Cisplatin $(40 \mathrm{mg} / \mathrm{m} 2)$ for 6 weeks of the radiotherapy course. The patient tolerated the whole treatment course with no unintended breaks. Because of fast primary tumor and nodal regression, observed after 20 Gy (fraction 10th) with CBCT imaging, replanning was required and a new thermoplastic mask, CT scans, contouring and treatment plan were done. The observed early radiation side effects according to CTCAE V.4, were grade 2 dermatitis, grade 2 mucositis, and grade 2 dysgeusia and weight lost. For dealing with mucositis and dermatitis symptomatic treatment was given and complete resolution was achieved. After treatment completion, the patient was evaluated monthly for the first 3 months and then every 2 months till the end of the first year by ENT specialist. The follow-up included physical exam and endoscopic investigation at each appointment. The follow up imaging consisted of MRI, and PET/CT at 3, 6, 9 and 12 months after treatment completion or when clinically indicated. MRI at third month reported suspicion for the residual formation. PET/CT at 6 month reported - no findings for the residual formation or distance metastases -complete response. MRI at 9 - month - complete response. (fig. 2). PET/CT at 12 month- complete response. No active disease or distant metastases (fig.1). Except grade 2 dry mouth at follow up, no late toxicity is observed.

\section{Discussion}

In 2010 worldwide NPC leaded to 65,000 deaths worldwide compared to 45,000 in 1990. (5). In Bulgaria highest incidence in males is reported at age 50-59 years old and in females at age 45-54 years old. Incidence rate for a long period from 1981 to 
2009 was $0.9 / 100000$ in males and $0.4 / 100000$ in females. Advanced stages T3-4 were diagnosed in $83 \%$ of the patients. Late diagnosis was due to patients unwillingness for medical consult (42\%), the general practitioner expertise $(11 \%)$, the ENT specialist expertise $(23 \%)$ and other professionals expertise-dentist or neurologist (24\%) (6) The 5 years overall survival in patients with NPC is estimated to be $72 \%$ for I stage, $64 \%$-II stage; $62 \%$ - II stage and $38 \%$ for IV stage. (7). The difficulties with diagnosis and the advanced stage of presentation are related to the symptoms which are not so specific for the disease. Correct staging of NPC is the most important factor for decision making for treatment. Different imaging methods can give a different information for disease staging, especially MRI for the local tumor invasion and PET/CT scan for the distant metastases. Extension of NPC, into the skull base is well demonstrated on MRI, and it is the most precise and accurate imaging for the detection of NPC and subclinical forms of the NPC (8), The sensitivity of CT with i.v. contrast and PET/CT for skull base invasion, and intracranial extension of NPC is low (9), which we proved in current study.

Specificity and sensitivity of MRI is much higher than that of SPECT-CT and CT computed tomography (CT) in detecting skull base invasion in NPC patients and the most common used imaging modality CT scan is reported to be the less sensitive (10). Positron emission tomography (PET) is not so sensitive for detection of the primary tumor, but we get a more information for the primary tumor, $\mathrm{N}$ and $\mathrm{M}$ staging, and evaluation of treatment response $(11,12)$, like we described in our study.

MRI is a valuable imaging modality for correct staging of the tumor, and also for contouring the target volumes in radiotherapy treatment planning process, and nowadays is routinely incorporated in the planning process for the head and neck cancer $(13,14)$. We have integrated itin our is institutional protocol at UH "St. Marina” - Varna.
MRI has very good resolution of the soft tissue of the head and neck, enables accurate gross tumor definition which makes it the preferred imaging mode, used in treatment planning of NPC (15). In the presented case if the contouring of the target volumes was done without the use of MRI images then the tumor, that had invaded intracranially was not going to be irradiated which could reflect in bad local control and relevantly poor survival of the patient. So, with the present case report we would like to stress the value of MRI in NPC, not only for staging but for precise target delineation in radiotherapy workflow, with direct contribution to survival.

The modern radiotherapy planning systems allow fusion between the planning CT for calculating the radiation dose, and MRI data set in treatment position for improving contouring of soft-tissue like tumor and involved lymph nodes and organs at risk like chiasm, brain, brainstem, intraorbital tissue, and skull base invasion in NFC. That is very important for correct delivering the recommended total dose of 60-70 Gy, because of the close tumor location to healthy organs at risk, which tolerance doses are below the treatment dose required.

Age of the patient, proximity of brainstem; optic nerves and optic chiasma, good response after second week, concurrent weekly Cisplatin which radiobiologically increased effectiveness of the delivered total dose and evaluation of the benefits and risks for the patient quality of life, overall survival and tumor response, were reasons for delivering 64 Gy in gross tumor volume (GTV).

\section{Conclusion}

MRI findings in NPC can upstage the tumor, especially in patients with perineural and scull base invasion. The implementation of MRI in radiotherapy treatment planning is of utmost importance due to precise and correct definition of GTV, especially in case of intracranial invasion. MRI staging should be part of the initial staging and follow up algorithm in all patients with NPC. 

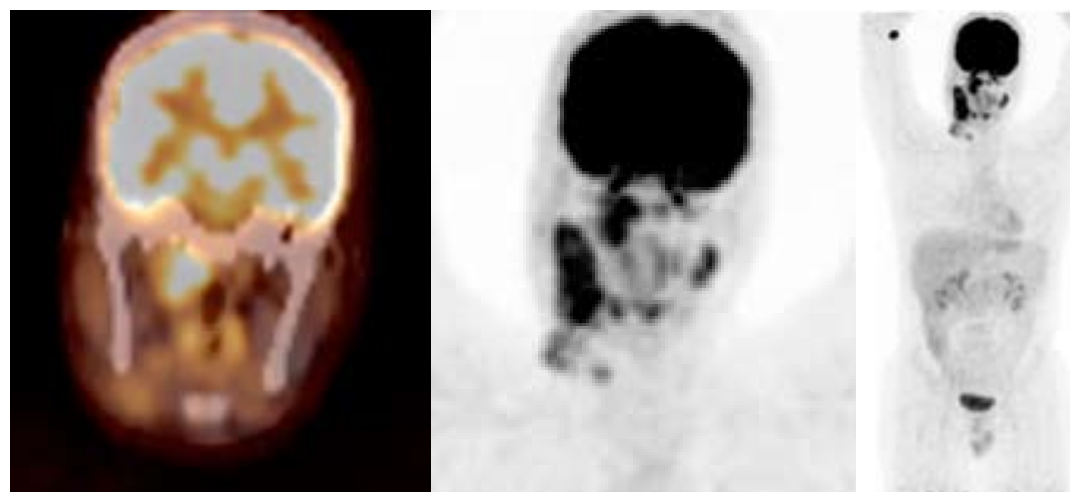

Staging PET-CT
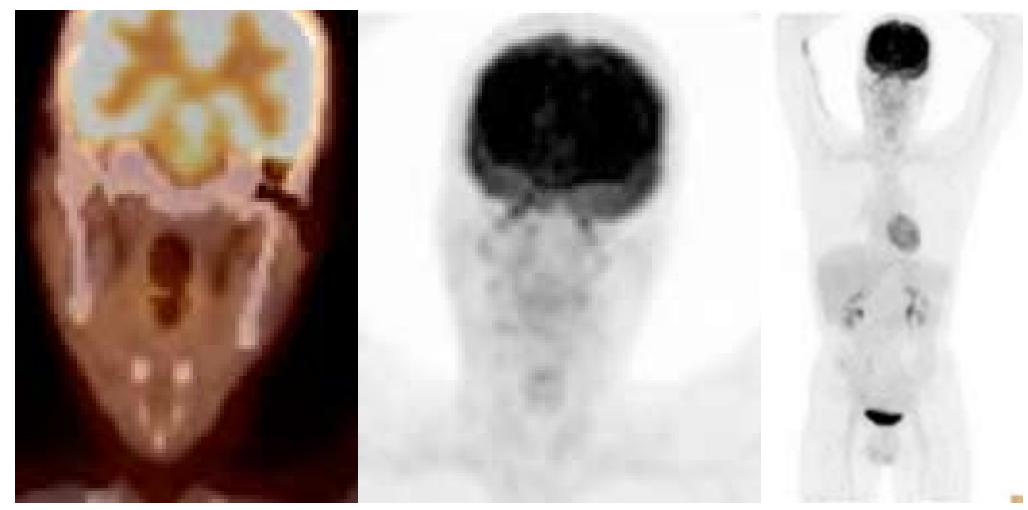

Follow up PET-CT at 1 year

Fig. 1. Staging PET CT, where no brain invasion is detected and the complete response at 1 year
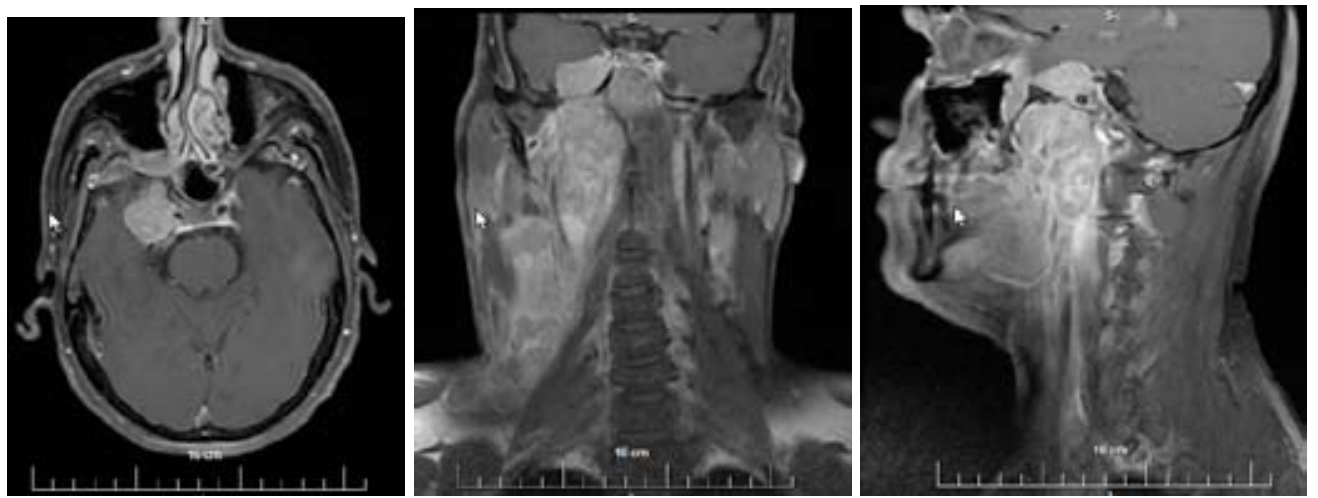

Staging MRI
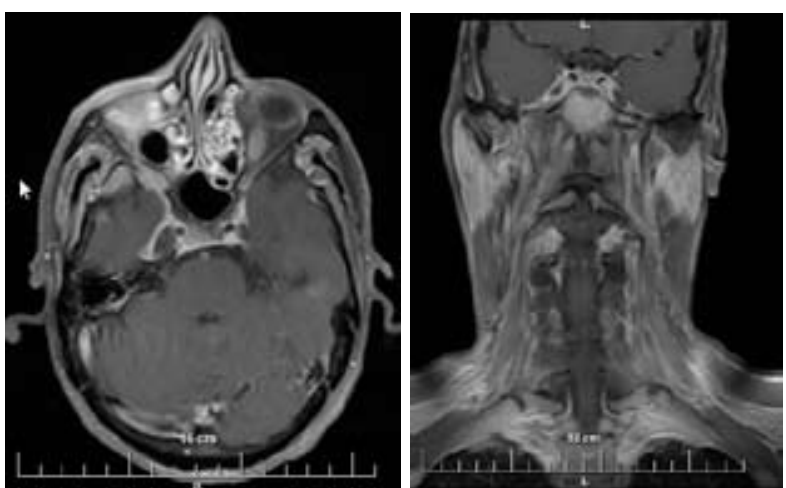

Follow up MRI at 9 month

Fig. 2. Staging MRI detected intracranial brain invasion and the complete response seen at 9 month 

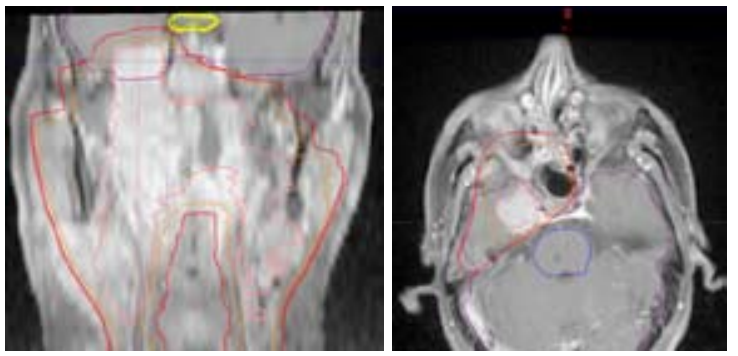

Fig. 3. Contouring target volumes based on MRI, where in close proximity optic chiasm, optic nerves and brainstem are seen

\section{References:}

1. Young L. S., and Dawson C. W. 2014.Epstein Barr virus and nasopharyngeal carcinoma. Chin. J. Cancer 33:581-590.

2. Raab Traub N. 2002. Epstein Barr virusin pathogenesis of NPC. Semin. Cancer Biol. 12:431-441.

3. Bulgaria National Cancer Registry 2016

4. Brennan, Bernadette (June 26, 2006). "Nasopharyngeal carcinoma”. Orphanet Journal of Rare Diseases. 1:23: 1-5.

5. Lozano, R (Dec 15, 2012). "Global and regional mortality from 235 causes of death for 20 age groups in 1990 and 2010: a systematic analysis for the Global Burden of Disease Study 2010”. Lancet. 380 (9859): 2095-128.

6. Desislava Skerleva and Stefan Stoyanov. Nasopharyngeal carcinoma-causes for delay of diagnosis. Deutsche Gesellschaft für Hals-Nasen Ohren-Heilkunde, Kopf- und Hals-Chirurgie. 84. Jahres versammlung der Deutschen Gesellschaft für Hals-Nasen-Ohren-Heilkunde, Kopfund Hals-Chirurgie. Nürnberg, 08. 12.05.2013. Düsseldorf: GermanMedical Science GMS Publishing House; 2013. Doc13hnod207

7. Survival Rates for Nasopharyngeal Cancer by Stage, NCS, 20.12.2017

8. King AD, Vlantis AC, Bhatia KS, etal. Primary nasopharyngeal carcinoma: diagnostic accuracy of MR imaging versus that of endoscopy and endoscopic biopsy. Radiology 2011; 258:531-537

9. Lau J. S., Yeo W., Chan Y. L. et al. 2004. Intracranial metastasis from nasopharyngeal carcinoma: report of three cases and review of literature. J. Clin. Oncol. 22, no. 14_suppl:5608.

10. Zhang S. X., Han P. H., Zhang G. Q., et al. 2014. Comparison ofSPECT/CT, MRI and CT in diagnosis of skull base bone invasion in nasopharyngeal carcinoma. Biomed. Mater. Eng. 24:1117-1124.

11. Zhu A., Lee D., and Shim H. 2011. Metabolic PET imaging in cancer detection and therapy response. Semin. Oncol. 38:55-69.

12. Mohandas A., Marcus C., Kang H. et al. 2014. FDG PET/CT in the management of nasopharyngeal carcinoma. AJR Am. J. Roentgenol. 203: W146-W157.

13. „Initiative for Vaccine Research (IVR): Viral cancers“. World Health Organization. Retrieved 2 October 2012.

14. Dirix P, Haustermans K, Vandecaveye V. The value of magnetic resonance imaging for radiotherapy planning. Seminars in radiation oncology. 2014;24(3):151-9.

15. Lai V, Khong PL. Updates on MR imaging and F-FDG PET/CT imaging in nasopharyngeal carcinoma. Oral Oncol 2013;50:539-48. 